\title{
Eurostudia
}

\section{La régulation du port des signes religieux à l'école en Allemagne entre acteurs politiques et judiciaires}

\section{Sylvie Toscer-Angot}

Volume 13, numéro 1-2, 2018-2019

Cultures en contact, entre régulations et représentations

URI : https://id.erudit.org/iderudit/1064492ar

DOI : https://doi.org/10.7202/1064492ar

Aller au sommaire du numéro

\section{Éditeur(s)}

Le Centre canadien d'études allemandes et européennes

ISSN

1718-8946 (numérique)

Découvrir la revue

Citer cet article

Toscer-Angot, S. (2018). La régulation du port des signes religieux à l'école en Allemagne entre acteurs politiques et judiciaires. Eurostudia, 13(1-2), 139-156. https://doi.org/10.7202/1064492ar

\section{Résumé de l'article}

$\mathrm{Au}$ cours des dernières décennies, la question de la régulation de la diversité religieuse croissante de la société et celle des libertés à accorder sur le plan religieux aux minorités dans la sphère publique, et notamment dans le système éducatif, ont fait l'objet de nombreuses discussions en Allemagne. L'une des questions les plus débattues concernait le port du foulard islamique par les enseignantes à l'école. En septembre 2003, la Cour constitutionnelle fédérale a énoncé que le Bade-Wurtemberg ne pouvait proscrire le port du foulard en l'absence de fondement législatif, mais que les législateurs des Länder pouvaient l'approuver ou l'interdire. Entre 2004 et 2006, huit Länder ont voté des lois interdisant le foulard islamique à l'école ou dans la fonction publique conformément au principe de neutralité de l'État. En 2015, la Cour constitutionnelle fédérale a statué qu'une interdiction globale était excessive et contrevenait à la liberté religieuse des enseignantes. L'objet de cette contribution est de mettre en évidence la gouvernance multiniveaux en matière de régulation de la diversité religieuse, l'entrecroisement des décisions judiciaires et politiques et l'influence croissante des différentes juridictions sur le législateur.
Tous droits réservés $@$ Le Centre canadien d'études allemandes et européennes, 2019
Ce document est protégé par la loi sur le droit d'auteur. L'utilisation des services d’Érudit (y compris la reproduction) est assujettie à sa politique d'utilisation que vous pouvez consulter en ligne.

https://apropos.erudit.org/fr/usagers/politique-dutilisation/ 
La régulation du port des signes religieux à l'école en Allemagne entre acteurs politiques et judiciaires

\author{
Sylvie Toscer-Angot \\ Université Paris-Est Créteil Val-de-Marne; \\ GSRL (EPHE-CNRS), PSL Research University
}

\title{
Résumé
}

Au cours des dernières décennies, la question de la régulation de la diversité religieuse croissante de la société et celle des libertés à accorder sur le plan religieux aux minorités dans la sphère publique, et notamment dans le système éducatif, ont fait l'objet de nombreuses discussions en Allemagne. L'une des questions les plus débattues concernait le port du foulard islamique par les enseignantes à l'école. En septembre 2003, la Cour constitutionnelle fédérale a énoncé que le Bade-Wurtemberg ne pouvait proscrire le port du foulard en l'absence de fondement législatif, mais que les législateurs des Länder pouvaient l'approuver ou l'interdire. Entre 2004 et 2006, huit Länder ont voté des lois interdisant le foulard islamique à l'école ou dans la fonction publique conformément au principe de neutralité de l'État. En 2015, la Cour constitutionnelle fédérale a statué qu'une interdiction globale était excessive et contrevenait à la liberté religieuse des enseignantes. L'objet de cette contribution est de mettre en évidence la gouvernance multiniveaux en matière de régulation de la diversité religieuse, l'entrecroisement des décisions judiciaires et politiques et l'influence croissante des différentes juridictions sur le législateur. 


\begin{abstract}
In the last few decades, there have been many debates in Germany over how to deal with an increasingly diverse society and over how much freedom should be given to minorities to express their religious identity in the public realm and especially within the educational system. One of the most important debates was about female Muslim teachers wearing a headscarf at school. In September 2003 the Constitutional Court ruled that Baden-Wurttemberg was wrong to ban a Muslim teacher from teaching, because the ban "has no legal basis in Baden-Wuerttemberg's law". The Court's ruling declared that German federal states are free to ban or approve Muslim headscarves in state schools. Between 2004 and 2006, eight federal states passed laws prohibiting Muslim teachers from wearing a headscarf at school or in civil service in accordance to the principle

of state neutrality. In 2015 a new Constitutional Court's ruling declared that "a statutory prohibition on expressing religious beliefs at the Land level [...] by outer appearance in an interdenominational comprehensive state school based on the mere abstract potential to endanger the peace at school or the neutrality of the state is disproportionate [...]". This paper aims at giving a clearer insight into the multilevel governance of religious diversity, into the judicial and legislative interactions and the increasing influence of the judiciary on legislative decision-making.
\end{abstract}

\title{
Introduction
}

Depuis la fin du XXe siècle, la société allemande connaît une pluralisation religieuse de plus en plus affirmée, liée à l'accroissement des flux migratoires, dans un contexte d'individualisation des croyances et des pratiques religieuses (Bader 2007; Casanova 2007; Bramadat et Koenig 2009). Cette diversification du paysage religieux donne lieu à des demandes de reconnaissance et de visibilité, mettant en jeu la question de la liberté religieuse et du droit à la différence religieuse dans les institutions publiques d'État et bousculant les principes normatifs sur lesquels se fondait la régulation publique du fait religieux (Reuter et Kippenberg 2010; Reuter 2014; Gerster et al. 2018). 
Les débats sur l'extériorisation des signes religieux pour le personnel éducatif dans les écoles publiques en Allemagne, et tout particulièrement sur le port $\mathrm{du}$ foulard islamique (hijāb) ${ }^{1}$, ont ainsi connu de nombreux rebondissements politiques et judiciaires depuis l'émergence de la première « affaire du foulard » en 1998 dans le Bade-Wurtemberg. La décision de la Cour constitutionnelle fédérale du 27 janvier $2015^{2}$, qui autorise largement le port du foulard islamique par les enseignantes à l'école, semble marquer un tournant décisif, voire un revirement de jurisprudence, par rapport à la décision de la Cour constitutionnelle du 24 septembre $2003^{3}$ qui avait laissé aux Länder le soin de légiférer sur la question. Huit d'entre eux avaient alors adopté des lois prohibitives en la matière. À l'inverse de la France qui a durci sa législation en matière de signes religieux ces dernières années, la décision de la juridiction suprême allemande de janvier 2015, très favorable à l'expression publique des signes religieux pour le personnel enseignant, a eu pour effet d'assouplir la plupart des lois d'interdiction sur le port de signes religieux à l'école, entrées en vigueur dans de nombreux Länder à partir de 2004.

L'objet de cette contribution est de déterminer, à partir de l'exemple du port $\mathrm{du}$ foulard par les enseignantes, comment s'opère l'entrecroisement des niveaux de régulation entre le Bund et les Länder en matière de régulation de la diversité religieuse. Il s'agit donc d'étudier la complexité des décisions mettant en jeu une pluralité d'acteurs politiques et d'acteurs judiciaires, qui interagissent et peuvent

\footnotetext{
${ }^{1}$ Nous parlons ici de foulard et non de voile, car c'est le terme Kopftuch (foulard) qui est exclusivement utilisé en Allemagne, même si selon les islamologues le terme « voile » est plus adéquat en référence au mot arabe $h i j a ̈ b$. Sur la question du hijäb, voir les versets du Coran invoqués $(24,30-31 ; 33,32-33 ; 33,53 ; 33,59)$ qui ne sont pas eux-mêmes univoques ou précis.

2 Voir la décision de la première chambre de la Cour constitutionnelle fédérale du 27 janvier 2015 : BVerfG, Beschluss des Ersten Senats vom 27. Januar 2015 - 1 BvR 471/10 - Rn. (131); http://www.bverfg.de/e/rs20150127 1bvr047110.html.

${ }^{3}$ Voir le jugement de la deuxième chambre de la Cour constitutionnelle fédérale du 24 septembre 2003 : BVerfG, Urteil des Zweiten Senats vom 24. September 2003 - 2 BvR 1436/02 - Rn. (1-138); http://www.bverfg.de/e/rs20030924 2bvr143602.html.
} 
se contredire. Comment s'articulent normes législatives et jurisprudentielles dans un pays fédéral où les questions religieuses relèvent des compétences des Länder? La décision des juges de Karlsruhe de janvier 2015 révèle-t-elle un affaiblissement du rôle des acteurs politiques au profit d'un renforcement de celui des acteurs judiciaires?

\section{Le cadre juridique et le recours à la justice pour des demandes de nature religieuse dans le cadre scolaire}

Le champ scolaire, fortement marqué par la tradition chrétienne en Allemagne, confronte celles et ceux qui en font partie - comme agents ou comme usagers - à la question des appartenances confessionnelles. Il se révèle être un lieu de cristallisation des conflits relatifs à la visibilité et à la reconnaissance du religieux dans sa diversité, étudiés ici au prisme du port du foulard par les enseignantes de confession musulmane dans le cadre scolaire.

Contrairement au cas de la France où les croyances et les pratiques religieuses n'ont pas droit de cité à l'école en vertu de la laïcité, celles-ci peuvent franchir sans difficulté le seuil de l'école en Allemagne (cf. Le Goff 2003). La liberté religieuse inscrite à l'article 4 de la Loi fondamentale ${ }^{4}$, qui comprend la liberté de croire, de professer sa religion et de la pratiquer, ne pose guère de problèmes particuliers pour les élèves au sein de l'école. Les tenues vestimentaires conformes à des croyances religieuses sont ainsi autorisées, comme en témoigne la possibilité pour les écolières de confession musulmane de porter un foulard ${ }^{5}$. Quant au cours

\footnotetext{
${ }^{4}$ Voir l'article 4 de la Loi fondamentale : « (1) La liberté de croyance et de conscience et la liberté de professer des croyances religieuses et philosophiques sont inviolables. (2) Le libre exercice du culte est garanti. »

5 À ce jour, les seules exceptions concernent l'interdiction de la burka à l'école en Hesse (depuis 2011), en Basse-Saxe et en Bavière (depuis 2017).
} 
de religion, il s'agit de la seule matière scolaire bénéficiant d'une garantie constitutionnelle 6 .

Les premières demandes individuelles de dispense de cours mixtes d'éducation physique auprès des établissements scolaires ont émergé à la fin des années 1980. La demande de dérogation formulée en 1989, en Rhénanie du NordWestphalie, par un père pour sa fille de confession musulmane, âgée de douze ans, est alors rejetée par les autorités scolaires au motif qu'il n'est pas possible de refuser de participer au cours d'éducation physique pour une raison religieuse, la priorité étant donnée à l'obligation scolaire. Le tribunal administratif de Gelsenkirchen, saisi de l'affaire en 19907, rejette la demande de dispense pour motif religieux, estimant que l'élève peut trouver une tenue adaptée au cours de sport et à ses convictions ou que ses parents peuvent le cas échéant choisir un établissement proposant des cours d'éducation physique non mixtes. Après un rejet de la requête par la Cour administrative d'appel (Oberverwaltungsgericht) de Rhénanie du NordWestphalie, qui privilégie également l'obligation scolaire par rapport à la liberté religieuse, le jugement de la Cour administrative fédérale (Bundesverwaltungsgericht) de Leipzig de $1993^{8}$ clôt la procédure devant les juridictions administratives, en déclarant que l'établissement scolaire doit épuiser toutes les possibilités d'organisation à sa disposition pour permettre à l'élève de participer à un cours de sport conforme à ses convictions, mais que l'école est tenue de lui accorder une dérogation si une telle possibilité n'existe pas. Au terme d'une bataille de plusieurs années devant les juridictions administratives, le père obtient ainsi la possibilité de faire dispenser sa fille des cours mixtes d'éducation physique. Face à des exigences apparemment contradictoires, telles que la mission éducative

\footnotetext{
${ }^{6}$ Voir l'article 7, alinéa 3 de la Loi fondamentale.

${ }^{7}$ Voir le jugement du tribunal administratif de Gelsenkirchen du 26 juin 1991 : VG Gelsenkirchen, 26.06.1991 - 4 K 2015/90.

8 Voir le jugement de la Cour administrative fédérale de 1993: BVerwG, 25.08.1993 - 6 C 8/91; https://www.jurion.de/Urteile/BVerwG/1993-08-25/BVerwG-6-C-8 91.
} 
de l'institution scolaire, la liberté religieuse individuelle et le droit des parents d'éduquer leurs enfants selon leurs croyances et convictions personnelles, la Cour administrative fédérale a tranché, illustrant notamment la priorité accordée à la liberté religieuse individuelle dans l'arbitrage entre obligation scolaire et liberté religieuse. Si les juges ne sont guère enclins à accorder des dispenses de cours de sport ou de natation, ils attendent toutefois des établissements scolaires des « accommodements » permettant de concilier largement fréquentation de l'école et pratiques islamiques et évitant aux élèves d'être face à un conflit de conscience. Au cours des dernières années, plusieurs établissements scolaires ont refusé d'accorder à des élèves de confession musulmane des dispenses de cours de natation, tout en les autorisant à porter un burkini. À diverses reprises, des responsables politiques ont également déclaré que l'essentiel était la participation des jeunes filles de confession musulmane au cours de natation?

Depuis les années 1990, on observe un recours croissant à la justice pour des demandes individuelles de nature religieuse. Si la présence visible de l'islam à travers des signes religieux distinctifs n'est pas un problème pour les élèves, le port du foulard a commencé à faire débat pour les enseignantes vers la fin des années 1990.

\section{Les décisions de justice relatives au port du foulard islamique par des enseignantes 1998-2003}

Le premier conflit relatif au foulard islamique a vu le jour dans le BadeWurtemberg en 1998, marquant le début d'une saga judiciaire de plusieurs années qui a suscité un vaste débat à l'échelle nationale. Le port du foulard à l'école par des enseignantes de confession musulmane, dont la visibilité était perçue à

\footnotetext{
9 On peut citer par exemple les propos de Franziska Giffey, ministre fédérale de la Famille, invitée à s'exprimer sur le sujet par l'hebdomadaire Die Zeit à Hambourg, le 24 juin 2018 : « Le plus important est le bien des enfants, à savoir que tous apprennent à nager » (trad. de l'auteure).
} 
l'époque comme non légitime par la quasi-totalité des acteurs politiques, a été régulé entre 1998 et 2003 par des procédures judiciaires, avant d'être renvoyé vers les législateurs de chaque Land à l'issue de la décision de la Cour constitutionnelle de septembre 2003.

En juillet 1998, les autorités scolaires du Bade-Wurtemberg refusent de recruter dans la fonction publique Fereshta Ludin, une enseignante stagiaire de nationalité allemande et d'origine afghane qui refuse d'enlever son foulard en classe $^{10}$. Elles mettent ainsi en avant son "manque d'aptitude à enseigner » au motif que le port d'un foulard représente « une atteinte au principe de neutralité auquel est tenu tout représentant de l'État ${ }^{11}$. Quant à la ministre de l'Éducation du Bade-Wurtemberg, Annette Schavan (CDU), elle prend aussi clairement position contre le port du foulard, y voyant un « signe de différenciation culturelle et civilisationnelle ${ }^{12}$. S'estimant lésée dans son droit fondamental à la liberté religieuse ${ }^{13}$ et victime de discriminations de nature religieuse ${ }^{14}$, Fereshta Ludin décide de se pourvoir en justice. La requête de la plaignante est tout d'abord rejetée par le tribunal administratif de Stuttgart ${ }^{15}$, qui fait valoir que le port du foulard à l'école représente une violation du principe de neutralité confessionnelle de l'État qu'un agent est censé respecter, d'une part, et une atteinte à la liberté négative des

\footnotetext{
${ }^{10}$ Fereshta Ludin était enseignante en primaire et dans un établissement secondaire du premier cycle (Hauptschule).

11 Voir : Bescheid des Oberschulamts Stuttgart, 10 juillet 1998.

12 Voir : Annette Schavan, débat parlementaire du Land de Bade-Wurtemberg, 15 juillet 1998, Plenarprotokoll 12/51, 12. Wahlperiode, 51. Sitzung, p. 3984; $\underline{\text { https://www.landtag- }}$ bw.de/files/live/sites/LTBW/files/dokumente/WP12/Plp/12 0051 15071998.pdf\#page=3977.

${ }^{13}$ Fereshta Ludin se réfère alors notamment à l'article 4 , alinéas 1 et 2 de la Loi fondamentale.

${ }^{14}$ Elle se réfère également à l'article 33, alinéa 3 de la Loi fondamentale relatif à la non-discrimination dans la fonction publique pour des motifs de nature religieuse : "La jouissance des droits civils et civiques, l'admission aux fonctions publiques ainsi que les droits acquis dans la fonction publique sont indépendants de la croyance religieuse. Personne ne doit subir de préjudice en raison de son adhésion ou de sa nonadhésion à une croyance religieuse et philosophique ».

${ }^{15}$ Voir le jugement du tribunal administratif de Stuttgart du 24 mars 2000 : VG Stuttgart, 24.03.2000 - $15 \mathrm{~K}$ 532/99; https://www.jurion.de/Urteile/VG-Stuttgart/2000-03-24/15-K-532 99.
} 
élèves et au droit des parents d'éduquer leur enfant selon leurs croyances, d'autre part. La requête est également rejetée par la Cour administrative fédérale, qui confirme dans son jugement du 4 juillet $2002^{16}$ - en vertu de l'impératif de neutralité de l'État et au nom de la liberté religieuse négative des parents et des élèves - l'interdiction du port du foulard en classe, susceptible de porter atteinte aux croyances des élèves déclarant une autre appartenance religieuse que l'islam.

Fereshta Ludin, dont la plainte est rejetée par les différentes instances de la juridiction administrative, porte finalement l'affaire devant la Cour constitutionnelle fédérale, qui rend son jugement le 24 septembre 200317. Ce dernier précise que le port du foulard relève de la protection de la liberté de conscience et de religion et reconnaît - en l'absence d'interdiction législative explicite dans le Land concerné - le droit de la requérante à porter un foulard à l'école. Au nom de la liberté religieuse et de la non-discrimination de la plaignante garanties par la Loi fondamentale, les juges de Karlsruhe annulent le jugement de la Cour administrative fédérale du 4 juillet $2002^{18}$. La décision du 24 septembre 2003 n'est pas dénuée d'ambiguïté toutefois, dans la mesure où elle défend la liberté de manifester une appartenance religieuse au sein de l'institution scolaire, tout en déclarant qu'une interdiction du port du foulard est possible sur une base législative ${ }^{19}$, laissant ainsi la porte ouverte à une interdiction par les Länder. Dès

\footnotetext{
16 Voir le jugement de la Cour administrative fédérale du 4 juillet 2002 : BVerwG, 04.07.2002 - 2 C 21-01; http://www.bverwg.de/entscheidungen/pdf/240604U2C45.03.0.pdf.

${ }_{17}$ Voir le jugement de la deuxième chambre de la Cour constitutionnelle fédérale du 24 septembre 2003 : BVerfG, Urteil des Zweiten Senats vom 24. September 2003 - 2 BvR 1436/02 - Rn. (1-138); http://www.bverfg.de/e/rs20030924 2bvr143602.html.

${ }^{18}$ Le jugement de la Cour administrative fédérale du 4 juillet 2002 précisait qu'une enseignante ne pouvait conserver son foulard en classe, car elle portait atteinte au droit des parents à envoyer leurs enfants dans des écoles conformes à leurs croyances ou à les tenir éloignés de croyances qu'ils jugeraient fausses ou nuisibles.

${ }^{19}$ Voir supra le jugement de la Cour constitutionnelle du 24 septembre 2003. Voir également le commentaire du juriste Hans Michael Heinig sur cette décision de la Cour constitutionnelle fédérale « Religionsfreiheit oder Neutralitätsgebot? », 28 juin 2005;
} 
lors, la question est renvoyée au champ politique, et plus particulièrement au législateur.

Trois juges de la Cour constitutionnelle exprimèrent à l'époque une opinion dissidente (Jentsch, Di Fabio et Mellinghoff), insistant sur la difficulté à concilier le principe de liberté religieuse positive d'une enseignante et l'impératif de neutralité confessionnelle de l'État. Considérant comme prioritaire le devoir de loyauté visà-vis de l'État constitutionnel auquel est soumis tout fonctionnaire, ils en déduisirent que le port du foulard en classe n'était pas conciliable avec l'impératif de neutralité incombant à un fonctionnaire ${ }^{20}$.

\section{Les prolongements législatifs du jugement de la Cour constitutionnelle de 2003}

Dès octobre 2003, bon nombre de Länder annoncent leur intention de légiférer pour interdire aux enseignantes le port du foulard. Le dessaisissement des juges au profit des législateurs conduit ainsi huit Länder à voter, entre 2004 et 2006, des lois proscrivant les signes religieux ou philosophiques pour les représentants de l'État dans les écoles publiques ou dans la fonction publique, avec des inflexions parfois significatives ${ }^{21}$. Parmi ces huit Länder, on peut distinguer Brême et Berlin (Toscer-Angot 2013), qui ont interdit le port de signes religieux pour toutes les confessions sans aucune exception. Contrairement aux recommandations de l'arrêt de la Cour constitutionnelle de septembre 2003 qui défendait le principe d'une égalité de traitement entre les religions, les six autres Länder (Bade-Wurtemberg,

http://www.bpb.de/themen/SQH1C3,0,0,Religionsfreiheit oder Neutralit\%EF\%BF\%BDtsgebot.html.

Hans Michael Heinig faisait valoir dès 2005 que l'interdiction exclusive du foulard islamique dans certains Länder, signe d'une religion spécifique, était problématique.

${ }^{20}$ Voir l'opinion dissidente des trois juges : BverfG, Urteil des Zweiten Senats vom 24. September 2003 - 2 BvR 1436/02 - paragraphes 74-138; http://www.bverfg.de/e/rs20030924 2bvr143602.html.

${ }^{21}$ Sur l'ensemble des lois d'interdiction votées dans les différents Länder, voir le site de l'Institut européen de droit constitutionnel de Trèves (Institut für europäisches Verfassungsrecht): https://www.uni-trier.de/index.php?id=24373\#c48086. 
Sarre, Hesse, Bavière, Rhénanie du Nord-Westphalie et Basse-Saxe) ont fait le choix d'un traitement différencié des religions et ils ont assorti la prohibition des signes religieux pour le personnel éducatif de dérogations particulières pour les signes relevant de la culture «chrétienne occidentale», rappelant que le christianisme était le fondement de l'enseignement scolaire ${ }^{22}$. Dans ces six Länder, les législations adoptées entre 2004 et 2006 ont mis en place de manière implicite des régimes de reconnaissance, renonçant de fait au principe de neutralité confessionnelle. Pour reprendre les termes de Julie Ringelheim, on peut parler ici de «choix préférentiel» (Ringelheim 2014: 181) en matière religieuse, c'est-à-dire d'une préférence marquée pour le christianisme. Du reste, en Hesse et à Berlin, les lois $\mathrm{d}$ 'interdiction des signes religieux ne s'appliquent pas seulement au personnel de l'institution scolaire, mais à la fonction publique dans son ensemble (Toscer-Angot 2013).

Quant aux huit autres Länder (Hambourg, Schleswig-Holstein, RhénaniePalatinat et les cinq nouveaux Länder), ils n'ont pas adopté de loi ou de réglementation sur la question du foulard. Le nombre très faible de musulmans présents dans les nouveaux Länder peut expliquer l'absence de législation en la matière, tandis que les projets de loi visant à interdire aux enseignantes le port $\mathrm{du}$ foulard, déposés dans le Schleswig-Holstein et dans le Land de Rhénanie-Palatinat, n'ont guère connu de succès du fait des coalitions au pouvoir. Quant à Hambourg, il est le seul Land gouverné à l'époque par des chrétiens-démocrates à ne pas avoir présenté de projet de loi relatif à l'interdiction du foulard à l'école ${ }^{23}$.

\footnotetext{
22 Dans la loi scolaire modifiée de Rhénanie du Nord-Westphalie (§57, alinéa 4) du 13 juin 2006, il est question des "signes représentant les traditions ou valeurs éducatives et culturelles chrétiennes et occidentales».

${ }^{23}$ L'influence du maire de Hambourg, Ole von Beust, un responsable chrétien-démocrate libéral, favorable aux minorités, n'y est probablement pas étrangère. Au nom de la liberté religieuse, la majorité des députés de Hambourg s'est prononcée clairement contre toute loi prohibitive, préférant traiter de possibles litiges
} 


\section{La décision de la Cour constitutionnelle fédérale du 27 janvier 2015}

La période qui sépare la décision de la Cour constitutionnelle fédérale de septembre 2003 de celle de janvier $2015^{24}$ a été ponctuée - à la suite des législations prohibitives adoptées dans les différents Länder - par de nombreux jugements de tribunaux du travail qui ont rejeté la demande de port du foulard par des enseignantes ou membres de la communauté éducative.

La décision de la Cour constitutionnelle fédérale du 27 janvier 2015 fait suite aux recours de deux plaignantes contre l'article 57 alinéa 4 de la loi scolaire de Rhénanie du Nord-Westphalie du 13 juin 200625, interdisant au personnel enseignant «toute manifestation extérieure de ses convictions politiques, religieuses, philosophiques ou autres, de nature à mettre en danger ou perturber la neutralité du Land vis-à-vis des élèves et de leurs parents, ou la paix scolaire ${ }^{26}$ et prohibant « tout comportement extérieur susceptible de donner l'impression aux élèves ou aux parents qu'une enseignante ou un enseignant porte atteinte à la dignité humaine, à l'égalité des droits au sens de l'article 3 de la Loi fondamentale, aux libertés fondamentales et à l'ordre démocratique libéral ${ }^{27}$. Les requérantes, deux femmes de confession musulmane et de nationalité allemande, l'une

\footnotetext{
au cas par cas au niveau local. Des enseignantes ont ainsi continué à porter un voile en classe à Hambourg après 2003.

${ }^{24}$ Voir la décision de la première chambre de la Cour constitutionnelle fédérale du 27 janvier 2015 : BVerfG, Beschluss des Ersten Senats vom 27. Januar 2015 - 1 BvR 471/10 - Rn. (1-31); http://www.bverfg.de/e/rs20150127 1bvr047110.html).

${ }^{25}$ La loi scolaire de Rhénanie du Nord-Westphalie du 15 février 2005 a été modifiée dans un sens prohibitif le $13 \quad$ juin 2006: https://www.unitrier.de/fileadmin/fb5/inst/IEVR/Arbeitsmaterialien/Staatskirchenrecht/Deutschland/Kopftuchverbot/NR W Erstes Gesetz zur AEnderung des Schulgesetzes 13.6.2006.pdf.

${ }^{26}$ Voir § 57, alinéa 4 de la loi scolaire de Rhénanie du Nord-Westphalie de 2006.

${ }^{27}$ Ibidem
} 
éducatrice ${ }^{28}$ et l'autre enseignante de turc ${ }^{29}$, en poste dans des établissements publics de Rhénanie du Nord-Westphalie, portaient un foulard dans leur milieu professionnel depuis leurs recrutements respectifs en 1997 et en 2001. À la suite de la modification de la loi scolaire de Rhénanie du Nord-Westphalie du 13 juin 2006, leurs employeurs leur demandent de retirer leur foulard. L'une obtempère et remplace son foulard par une sorte de béret qui couvre ses cheveux et ses oreilles et par un pull à col roulé dissimulant son cou, mais elle est menacée de licenciement si elle ne modifie pas sa tenue, censée envoyer le même signal qu'un foulard. Quant à la seconde, qui refuse de retirer son foulard, elle est finalement licenciée en 2007. Estimant que ces décisions portent atteinte à leur liberté religieuse et sont contraires à la Loi fondamentale, les deux femmes saisissent la juridiction du travail. Leurs requêtes étant rejetées en première instance, en appel et en révision au motif que leur tenue est contraire au principe de neutralité de l'État, elles se tournent finalement vers la Cour constitutionnelle fédérale. Saisie de recours constitutionnels contre les jugements des différents tribunaux du travail, la première Chambre (Erster Senat) de la Cour constitutionnelle rend sa décision le 27 janvier 201530, à une majorité de six voix contre deux. Annulant les décisions précédentes des tribunaux du travail, elle fait valoir que le port de signes religieux à l'école, et en l'occurrence du foulard, par les enseignantes, est garanti par l'article 4 de la Loi fondamentale.

\footnotetext{
${ }^{28}$ Née en 1971 en Allemagne, d'origine turque, de confession musulmane et de nationalité allemande, cette éducatrice, en poste dans une école (Gesamtschule) de Rhénanie du Nord-Westphalie depuis 1997, a porté un foulard dans son établissement scolaire de 1997 à 2006.

29 Née en 1977, d'origine turque, de confession musulmane et de nationalité allemande, la seconde plaignante est une femme qui a fait des études menant au professorat. À partir de 2001, elle enseigne le turc comme contractuelle dans un établissement de Rhénanie du Nord-Westphalie, puis elle est titularisée.

${ }^{30}$ Voir la décision de la première chambre de la Cour constitutionnelle fédérale du 27 janvier 2015 : BVerfG, Beschluss des Ersten Senats vom 27. Januar 2015 - 1 BvR 471/10 - Rn. (1-31); http://www.bverfg.de/e/rs20150127 1bvr047110.html.
} 
Les juges de Karlsruhe se sont clairement prononcés en faveur d'une neutralité inclusive, ouverte à l'expression de la pluralité religieuse et ont ainsi interprété le port du foulard comme n'étant pas a priori en contradiction avec le principe de neutralité de l'État. Ils ont précisé, du reste, que l'article 57, alinéa 4 de la loi scolaire de Rhénanie du Nord-Westphalie était en contradiction avec l'article 3, alinéa 3 et avec l'article 33, alinéa 3 de la Loi fondamentale, qu'une interdiction globale des manifestations d'appartenance religieuse dans les écoles publiques était excessive et portait atteinte à la liberté de croyance et de religion, et que seul un danger concret mettant en cause la paix scolaire ou la neutralité de l'État pouvait justifier une interdiction des signes religieux. Les juges fédéraux ont estimé qu'en l'absence de prosélytisme ou de tentative avérée d'une enseignante de confession musulmane d'influencer les élèves, il n'était pas possible d'imposer une interdiction générale du foulard (Gaillet 2015; Heinig 2015; Rabault 2015). Ils ont ainsi rejeté l'argument selon lequel le simple port du foulard islamique mettait en question la paix scolaire ou la neutralité de l’État et réaffirmé la primauté des libertés et droits fondamentaux individuels, et notamment de la liberté religieuse. Réfutant toute conception de la neutralité de l'État comme exclusion des signes religieux, les juges de Karlsruhe ont défendu « une attitude d'ouverture favorisant la liberté de croyance pour toutes les confessions » ${ }^{31}$ et ils ont considéré le port du foulard comme le simple reflet de la pluralité religieuse de la société, tout en précisant qu'une telle tenue ne portait pas atteinte à la neutralité de l'État. À travers cette décision, les juges majoritaires de la juridiction suprême ont voulu donner un signal positif en faveur du traitement égalitaire des religions et de la diversité religieuse. Selon la juriste Aurore Gaillet, la décision de 2015 «s'apparente à un plaidoyer pour une société plurielle» (Gaillet 2015 : 1404).

\footnotetext{
${ }^{31}$ Voir la décision de la première chambre de la Cour constitutionnelle fédérale du 27 janvier 2015 : BVerfG, Beschluss des Ersten Senats vom 27. Januar 2015 - 1 BvR 471/10 - Rn. (1-31), par. 109-111; http://www.bverfg.de/e/rs20150127 1bvr047110.html.
} 


\section{Les conséquences du jugement de la Cour constitutionnelle fédérale de 2015 à l'échelle des Länder}

Loin de mettre fin à des conflits et des recours en justice, la décision de la Cour constitutionnelle de janvier 2015 soulève des incertitudes et des questions quant à son application. On peut en effet constater qu'elle n'est pas mise en œuvre de manière homogène dans les différents Länder ${ }^{32}$. L'autorisation pour les enseignantes de porter ou non un foulard au sein de l'école fait débat encore aujourd'hui. Plusieurs Länder ont assoupli leur législation ou du moins leurs pratiques. En juin 2015, la Rhénanie du Nord-Westphalie, par exemple, a supprimé de sa loi scolaire l'interdiction pour les enseignantes de porter un foulard à l'école et a opté pour un règlement au cas par cas, tout comme la Bavière, la Basse-Saxe, le Bade-Wurtemberg, etc.

Le maintien, malgré le jugement de la Cour constitutionnelle de 2015, de la loi de neutralité berlinoise restrictive de 2005, qui interdit à tous les agents de la fonction publique le port de signes religieux ostensibles, est révélateur des interférences de la gouvernance politique et de la régulation judiciaire. La révision de la loi de neutralité berlinoise divise aujourd'hui les différents partis de la coalition gouvernementale berlinoise au pouvoir ${ }^{33}$. Si le parti social-démocrate (SPD), principal partenaire du gouvernement dans le Land de Berlin, se montre farouchement hostile à toute révision de la loi de 2005, ses autres partenaires de coalition au pouvoir, les Verts et Die Linke, se sont exprimés en faveur d'une modification de la loi de neutralité berlinoise. La volonté affichée du SPD berlinois,

\footnotetext{
${ }^{32}$ Cette contribution s'appuie sur l'étude réalisée par les services de documentation du Bundestag en 2017 : Wissenschaftliche Dienste, Zur Situation Kopftuch tragender Lehrerinnen in ausgewählten Bundesländern, WD 8 - $3000 \quad$ - $3036 / 17, \quad 15 \quad$ septembre $\quad$ 2019; https://www.bundestag.de/blob/531764/49278f4c929edacd5c8e5858d69c6c67/wd-8-036-17-pdf-data.pdf, ainsi que sur des articles de presse, des débats parlementaires et des décisions de justice à l'échelle des Länder.

${ }^{33}$ Une coalition formée des sociaux-démocrates (SPD), des Verts et de Die Linke (gauche radicale) est au pouvoir à Berlin depuis 2016. Elle a succédé à une grande coalition SPD-CDU (2011-2016).
} 
soutenu par les chrétiens-démocrates berlinois, de ne pas modifier la loi de neutralité de $2005^{34}$ pourrait toutefois être mise en difficulté par des procédures judiciaires en cours, susceptibles de donner raison à des plaignantes de confession musulmane, illustrant le fait que « le droit constitue un enjeu important de luttes et de négociations entre ceux, gouvernants et gouvernés, qui cherchent à peser sur la définition de la place légitime du religieux dans l'espace public » (de Galembert et Koenig 2014 : 635). En mai 2017, la ministre chargée de l'éducation, Sandra Scheeres (SPD) ${ }^{35}$, a réaffirmé que le Land de Berlin ne ferait pas appel du jugement rendu en février 2017 par le tribunal du travail de Berlin, en vertu duquel la villeÉtat a dû verser 8700 euros de dédommagements à une enseignante berlinoise de confession musulmane. Cette dernière avait porté plainte contre le Land de Berlin, car elle s'estimait discriminée pour des motifs religieux, car elle n'avait pas été recrutée comme enseignante dans une école publique berlinoise en raison de son intention affichée de garder son foulard dans le cadre scolaire. À l'inverse, une enseignante berlinoise, qui voulait enseigner avec son foulard dans une école primaire, a été déboutée de sa plainte en 2018 par la juridiction du travail. Le cas de Berlin reste toutefois une exception, la quasi-totalité des Länder ayant infléchi leurs pratiques à la suite de la décision de la Cour constitutionnelle fédérale du 27 janvier 2015.

\section{Conclusion}

Les questions soulevées par la présence et la visibilité des signes religieux à l'école en Allemagne, qui mettent à l'épreuve le principe de neutralité religieuse et philosophique de l'État, révèlent le rôle décisif joué par la justice dans la régulation $\mathrm{du}$ religieux ou ce qu'on appelle encore la judiciarisation de la gouvernance

\footnotetext{
34 Susanne Memarnia, "Soll das Kopftuch verboten bleiben? », TAZ, 14 avril 2016; http://www.taz.de/!5292479/.

35 Sandra Scheeres (SPD) a été nommée ministre de l'Éducation, de la Jeunesse et de la Famille dans le Land de Berlin (Senatorin für Bildung, Jugend und Famillie) en décembre 2016.
} 
publique du religieux (de Galembert et Koenig 2014), ainsi qu'une intrication complexe des décisions politiques et judiciaires, le pouvoir juridictionnel influant largement aujourd'hui sur le pouvoir législatif. Le jugement de la Cour constitutionnelle de janvier 2015 apparaît comme un tournant qui témoigne d'une évolution normative en germe dans l'arrêt de 2003, une nouvelle interprétation du principe de neutralité confessionnelle de l'État étant désormais pleinement assumée par les juges constitutionnels. On voit ainsi se dessiner les nouveaux contours d'une neutralité favorable au pluralisme religieux et qui s'efforce de placer toutes les confessions sur un pied d'égalité, avec des variations d'un Land à l'autre concernant la question du port du foulard, révélatrices de visions du monde ou de conceptions de la neutralité confessionnelle de l'État concurrentes, liées à des identités et des particularismes affirmés et des contextes politiques ou culturels spécifiques. Du fait de sa dimension fédérale, indissociable de la culture juridique et politique allemande, le cas de l'Allemagne est particulièrement intéressant dans la mesure où il représente un laboratoire d'expérimentations variées et originales répondant à des contextes ou situations spécifiques. Hormis le cas de Berlin, exigeant la neutralité non seulement du personnel éducatif, mais aussi de tous les agents de la fonction publique, peu représentatif de l'ensemble de l'Allemagne, on constate qu'à l'inverse de l'évolution observable en France depuis quelques décennies (Valentin 2017), le périmètre du droit à la liberté religieuse individuelle et collective ne cesse de s'accroître outre-Rhin au fil des ans, laissant toujours plus de place aux religiosités individuelles dans l'espace public institutionnel. La priorité donnée au droit individuel à la liberté religieuse, placée au sommet de toutes les libertés depuis 1949 à la suite de l'expérience du national-socialisme, y représente ainsi une dynamique importante de la diversification des rapports sociaux. 


\section{Bibliographie}

Amir-Moazami, S. (2007). Politisierte Religion, Der Kopftuchstreit in Deutschland und Frankreich. Bielefeld, Transcript Verlag.

Bader, V. (2007). Secularism or Democracy? Associational Governance of Religious Diversity. Amsterdam, Amsterdam University Press.

Barras, A., F. Dermange et S. Nicolet (dir.). (2016). Réguler le religieux dans les sociétés libérales. Genève, Labor et Fides.

Becci, I., C. Monnot et O. Voirol (dir.). (2019). Pluralisme et reconnaissance. Rennes, PUR.

Bramadat, P. et M. Koenig (dir.). (2009). International Migration and the Governance of Religious Diversity. Montréal, McGill-Queen's University Press.

Casanova, J. (2007). « Immigration and the New Religious Pluralism. A European Union/United States Comparison ». Dans T. Banchoff (dir.), Democracy and the New Religious Pluralism. New York, Oxford University Press, p. 59-83.

Gaillet, A. (2015). « Port du voile par les enseignantes des écoles publiques : retour à Karlsruhe. Étude sur la décision de la Cour constitutionnelle allemande du 27 janvier 2015 ». AJDA, n²5, p. 1401-1407.

(de) Galembert, C. et M. Koenig. (2014). «Gouverner le religieux avec les juges. Introduction ». Revue française de science politique, vol. 64, no. 4, p. 631-645.

Gerster, D., V. van Melis et U. Wilhelms (dir.). (2018). Religionspolitik heute. Problemfelder und Perspektiven in Deutschland. Freiburg, Herder Verlag.

Heinig, H. M. et C. Walter (dir.). (2007). Staatskirchenrecht oder Religionsverfassungsrecht?. Tübingen, Mohr Siebeck.

Heinig, H. M. (2015). «Ein neues Kapitel in einer unendlichen Geschichte? Verfassungsrechtliche, prozessrechtliche und religionspolitische Anmerkungen zum Kopfbeschluss des Bundesverfassungsgerichts vom 27. Januar 2015 ». GOEDOC, Dokumenten- und Publikationsserver der GeorAugust-Universität, Göttinger E-Papers $\mathrm{zu}$ Religion und Recht 10, http://resolver.sub.uni-goettingen.de/purl/?webdoc-3952. 
Joppke, C. (2009). Veil. Mirror of Identities. Cambridge, Polity Press.

Joppke, C. et J. Torpey. (2013). Legal Integration of Islam: A Transatlantic Comparison. Cambridge (Mass.), Harvard University Press.

Konig, M. (2005). «Incorporating Muslim Migrants in Western Nation-States. A Comparison of the United Kingdom, France and Germany ». Journal of International Migration and Integration vol. 6, no. 2, p. 219-234.

Koenig, M. (2007). «Europeanising the Governance of Religious Diversity: An Institutionalist Account of Muslim Struggles for Public Recognition ». Journal of Ethnic and Migration Studies, vol. 33, n 6, p. 911-932.

Le Goff, A. (2003). «La neutralité religieuse de l'État et de l'école publique en France et en Allemagne ». Thèse de doctorat, Université Paris I, Faculté de droit.

Rabault, H. (2015). « Le droit des enseignantes à arborer le foulard (décision de la première chambre de la Cour constitutionnelle fédérale allemande du 27 janvier 2015) ». Revue française de droit constitutionnel, vol. 103, n³, p. 735744.

Reuter, A. et H. G. Kippenberg (dir.). (2010). Religionskonflikte im Verfassungsstaat. Göttingen, Vandenhoeck \& Ruprecht.

Reuter, A. (2014). Religion in der verrechtlichten Gesellschaft. Rechtskonflikte und öffentliche Kontroversen um Religion als Grenzarbeiten am religiösen Feld. CSRRW Bd. 5, Göttingen, Vandenhoeck \& Ruprecht.

Ringelheim, J. (2014). «Du voile au crucifix: la jurisprudence de la Cour européenne des droits de l'homme ». Dans V. Amiraux et D. Koussens (dir.), Trajectoires de la neutralité. Montréal, Presses de l'Université de Montréal, p. 171-184.

Toscer-Angot, S. (2013). «La loi de neutralité berlinoise du 27 janvier 2005 : une mise à l'épreuve du modèle national de régulation du religieux? ». Revue $d^{\prime}$ Allemagne et des pays de langue allemande, vol. $45, \mathrm{n}^{\circ} 2$, p. 471-482.

Valentin, V. (2017). « Laïcité et neutralité ». AJDA, n²4, p.1388-1394. 